\title{
On graphs whose orientations are determined by their Hermitian spectra
}

\author{
Yi Wang* Bo-Jun Yuan \\ School of Mathematical Sciences \\ Anhui University \\ Hefei, 230601, P. R. China \\ wangy@ahu .edu.cn \\ ybjmath@163.com
}

Submitted: Jun 11, 2020; Accepted: Aug 28, 2020; Published: Sep 18, 2020

(C) The authors. Released under the CC BY-ND license (International 4.0).

\begin{abstract}
A mixed graph $D$ is obtained from a simple graph $G$, the underlying graph of $D$, by orienting some edges of $G$. A simple graph $G$ is said to be ODHS (all orientations of $G$ are determined by their $H$-spectra) if every two $H$-cospectral graphs in $\mathcal{D}(G)$ are switching equivalent to each other, where $\mathcal{D}(G)$ is the set of all mixed graphs with $G$ as their underlying graph.

In this paper, we characterize all bicyclic ODHS graphs and construct infinitely many ODHS graphs whose cycle spaces are of dimension $k$. For a connected graph $G$ whose cycle space is of dimension $k$, we also obtain an achievable upper bound $2^{2 k-1}+2^{k-1}$ for the number of switching equivalence classes in $\mathcal{D}(G)$, which naturally is an upper bound of the number of cospectral classes in $\mathcal{D}(G)$. To achieve these, we propose a valid method to estimate the number of switching equivalence classes in $\mathcal{D}(G)$ based on the strong cycle basis, a special cycle basis introduced in this paper.
\end{abstract}

Mathematics Subject Classifications: 05C50

\section{Introduction}

All graphs considered in this paper are finite and loopless. A mixed graph $D_{G}$ (or $D$, for short) is obtained from a simple graph $G=(V, E)$, the underlying graph of $D_{G}$, by orienting a subset of $E(G)$. Accordingly, a mixed graph $D_{G}$ is also called an orientation of $G$, whose vertex set $V(D)$ is same as the vertex set $V(G)$ and whose edge set $E(D)$ consisting of two parts: undirected edge set $E_{0}(D)$ and directed edge (or arc) set $E_{1}(D)$.

${ }^{*}$ The corresponding author. Supported by National Natural Science Foundation of China (11771016, 11871073). 
Denote by $\mathcal{D}(G)$ the set of all mixed graphs with $G$ as their underlying graph. To distinguish undirected and directed edges, we denote an undirected edge between the vertices $u$ and $v$ by $\{u, v\}$ and a directed edge from $u$ to $v$ by $(u, v)$. If there is no confusion, we write $u v$ (or $v u$ ) instead of $\{u, v\}$ or $(u, v)$. We may view undirected edges in a mixed graph as bi-directed edges. From this point of view, a mixed graph is indeed a digraph.

The Hermitian adjacency matrix of $D$ is defined as $H(D)=\left[h_{u v}\right]$ with

$$
h_{u v}= \begin{cases}1 & \text { if }\{u, v\} \in E_{0}(D) \\ \mathrm{i} & \text { if }(u, v) \in E_{1}(D) \\ -\mathrm{i} & \text { if }(v, u) \in E_{1}(D) \\ 0 \quad & \text { otherwise }\end{cases}
$$

where $\mathrm{i}=\sqrt{-1}$. The eigenvalues (spectrum) of $H(D)$ are called the $H$-eigenvalues $(H$ spectrum) of $D$. The characteristic polynomial of $H(D)$ is denoted by $\Phi(D, \lambda)$. Two mixed graphs are called $H$-cospectral (or cospectral for short) if they have the same $H$ spectrum. These terminologies were introduced by Liu and $\mathrm{Li}$ [11] and independently by Guo and Mohar [8]. They investigated the properties of characteristic polynomials and $H$-eigenvalues of mixed graphs and studied cospectral problems for mixed graphs. A number of linear algebraic properties of undirected graphs have been shown to hold for mixed graphs.

Problems about existence of cospectral graphs have long history, motivated by a natural question: can we determine a graph by its spectrum? The problem traces back to more than 50 years ago and has received a lot of attention from researchers in recent years. Formally, an undirected graph $G$ is said to be determined by its spectrum ( $D S$, for short) if every graph cospectral to $G$ is isomorphic to $G$. For the background and known results about this problem, readers are referred to two surveys $[5,6]$ and the references therein.

The same question has yielded fewer results for mixed graphs. So far, mixed graphs that can be determined by their $H$-spectra have proven elusive. From a spectral analysis point of view, one of leading causes is that a large number of mixed graphs with the same underlying graph have an identical $H$-spectrum. Thus, researchers have turned to spectral determination of classes of switching equivalent mixed graphs rather than individual mixed graphs.

Let $G$ be a simple graph. Guo and Mohar [13] presented an operation on mixed graphs in $\mathcal{D}(G)$, called switching equivalence, that can preserve the $H$-spectrum of mixed graphs. Switching equivalence relationship naturally partitions $\mathcal{D}(G)$ into the equivalence classes. In this sense, mixed graphs in an equivalence class can be considered as having the same orientation. In addition, the number of switching equivalence classes of $\mathcal{D}(G)$, denoted by $n_{s}(G)$, can be used to estimate the number of mixed graphs in $\mathcal{D}(G)$ having the same $H$-spectrum. For instance, if $n_{s}(G)=1$, it implies that all mixed graphs in $\mathcal{D}(G)$ have the same $H$-spectrum. More on switching equivalence will be presented in Section 2.2.

Let $\mathscr{D}$ be a family of mixed graphs. Based on the above discussion, a mixed graph $D \in \mathscr{D}$ is said to be determined by its $H$-spectrum (or $D H S$, for short) on $\mathscr{D}$ if every graph in $\mathscr{D}$ cospectral to $D$ is switching equivalent to $D$. If $\mathscr{D}$ is the set consisting of 
all mixed graphs, we say that $D$ is DHS for short. Obviously, the property DHS is a stronger property than DS. Namely, if an undirected graph is DHS, then it is necessarily DS. However, the converse is not true as evidenced by the two graphs in Figure 2 of [13].

Recently, the $H$-spectrum of mixed graphs has been the subject of several publications. In addition to two fundamental papers [8, 11] mentioned above, Mohar [13] characterized all mixed graphs with rank 2, that is mixed graphs whose Hermitian adjacency matrices have rank 2, and investigated which mixed graphs with rank 2 are DHS. Wang et al. [18] and Tian and Wong [16] extended the research in [13] to mixed graphs with rank 3 and shown all connected mixed graphs with rank 3 are DHS on their family. Guo and Mohar [9] determined all mixed graphs whose spectral radii with respect to their Hermitian adjacency matrices are less than 2 and investigated which paths are DHS. Wissing and van Dam [19] constructed the first infinite family of connected (strongly) DHS mixed graphs. For further research works concerned with the $H$-spectra of mixed graphs, readers are referred to the literature $[2,3,4,7,10,20]$ and the references therein.

From a structural point of view, the structure of a mixed graph can be understood as two parts: the incidence structure, that is the structure of its underlying graph, and the orientation. Determining the former by spectrum is the familiar as "DS" problem and is widely studied. Orientations of graphs are closely related to colorings and flows of graphs. For instance, Minty [12] and Vitaver [17] proved that a graph $G$ is $k$-colorable if and only if $G$ has an orientation such that every cycle $C$ of $G$ contains at least $|C| / k$ edges in each of the two directions around the cycle. Steinberg and Younger [14] proved that a bridgeless graph $G$ admits a nowhere-zero 3-flow if and only if $G$ has a modular 3 -orientation. However, determining orientations of graphs by spectra has received less attention. This motivates us to discuss "DHS" on $\mathcal{D}(G)$. If for any $D \in \mathcal{D}(G)$, every graph in $\mathcal{D}(G) H$-cospectral to $D$ is switching equivalent to $D$, that is, $D$ is DHS on $\mathcal{D}(G)$, we say that orientations of $G$ are determined by their $H$-spectra (or $G$ is ODHS, for short). Clearly, if $G$ is not ODHS, then there are at least two mixed graphs (two switching equivalence classes) in $\mathcal{D}(G)$ which are not DHS.

The main purpose of our study is to determine which graphs are ODHS and which are not. The difficulty of this problem is that the switching equivalences of two mixed graphs are not well-understood. In Section 2, we will present a rough characterization of switching equivalences between two mixed graphs. In Section 4, we propose a valid method to estimate the number of switching equivalence classes in $\mathcal{D}(G)$ based on the strong cycle basis, and special cycle basis will be introduced in Section 3. As an application of the method, we prove that $n_{s}(G)$ is bounded above by $2^{2 k-1}+2^{k-1}$ for any connected graph $G$ whose cycle space has dimension $k$, which is naturally an upper bound of the number of cospectral classes in $\mathcal{D}(G)$. In Section 5, some necessary conditions of ODHS graphs are proposed. Using these results, we characterize all bicyclic ODHS graphs and construct infinitely many ODHS graphs achieving the upper bound given in Section 4 . 


\section{Preliminaries}

\subsection{Characteristic polynomials of mixed graphs}

Let $D$ be a connected mixed graph and $H(D)=\left[h_{i j}\right]$ be its Hermitian adjacency matrix. The value of a mixed walk $W: v_{1} v_{2} \ldots v_{s}$ is defined as $h_{12} h_{23} \ldots h_{s-1, s}$ and is denoted by $h(W)$. For a mixed cycle $C$, we assign a direction to $C$ before calculating its value. Obviously, if in one direction the value of a mixed cycle or a mixed path is $\alpha$, then in the reversed direction its value is $\bar{\alpha}$, the conjugate number of $\alpha$. A cycle $C$ is called positive (negative, resp.) if $h(C)=1(-1$, resp.). Positive or negative cycles are called real cycles, whose values are independent of their assigned direction. A cycle $C$ is called imaginary if $h(C) \in\{ \pm \mathrm{i}\}$.

For any cycle $C$ of a mixed graph $D$, we assign a direction for $C$. The operation is called cycle orientation of $D$. Let $D^{\prime}$ and $D^{\prime \prime}$ be two mixed graphs with the same underlying graph $G$. Taking a cycle in $D^{\prime}$, we obtain the corresponding cycle in $D^{\prime \prime}$ naturally. Hereafter, discussing two mixed graphs with the same underlying graph, we always suppose that they have the same cycle orientation, which is also called the cycle orientation of $G$. It is different to the terminology orientation of $G$ mentioned in Section 1 .

Let $D$ be a mixed graph of order $n$. The characteristic polynomial of $H(D)$ is denoted by

$$
\Phi(D, \lambda)=\lambda^{n}+c_{1} \lambda^{n-1}+\cdots+c_{n} .
$$

A Sachs subgraph is a subgraph only having $K_{2}$ or cycles as connected components. Let $S$ be a Sachs subgraph of $D$. For a given cycle orientation of $D$, the signature of $S$, denoted by $\sigma(S)$, is defined as $|f-b|$, where $f$ denotes the number of forward arcs and $b$ denotes the number of backward arcs in $S$. A Sachs subgraph $S$ is called basic if each cycle $C$ in $S$ is real. Equivalently, $C$ has an even number of directed edges, and $\sigma(C)$ is even for each cycle $C$ of $S$.

For a basic subgraph $B$, let $r(B)=\frac{1}{2} \sigma(B)$. It is clear that $(-1)^{r(B)}=\prod_{C \subset B} h(C)$, which is independent of the cycle orientation of $D$.

We state the following theorem from [11] in different format and notation.

Theorem 1. [11] Let $D$ be a mixed graph of order $n$ with characteristic polynomial $\Phi(D, \lambda)=\sum_{j=0}^{n} c_{j} \lambda^{n-j}$. Then

$$
c_{j}=\sum_{B}(-1)^{r(B)+s(B)} 2^{c(B)}=\sum_{B} \prod_{C \subset B} h(C)(-1)^{s(B)} 2^{c(B)},
$$

where the sum runs over all basic subgraphs $B$ of order $j$ in $D, s(B)$ is the number of connected components in $B$, and $c(B)$ is the number of cycles in $B$.

The authors of [21] proved the following theorem as a consequence of Theorem 1.

Theorem 2. [21] Let $D$ be a mixed graph and $v \in V(D)$. Let $\mathcal{C}_{v}$ be the set of all basic mixed cycles in $D$ containing $v$. Then

$$
\Phi(D, \lambda)=\lambda \Phi(D-v, \lambda)-\sum_{u v \in E(D)} \Phi(D-u-v, \lambda)-2 \sum_{C \in \mathcal{C}_{v}} h(C) \Phi(D-C, \lambda) .
$$




\subsection{The switching equivalence}

In $[8,13]$, the authors considered the so-called four-way switching operations, that preserve the $H$-spectrum of mixed graphs. We describe the definition in the matrix-theoretic language below.

Definition 3. [13] Let $D^{\prime}$ and $D^{\prime \prime}$ be two mixed graphs of order $n$ with the same underlying graph. A four-way switching is the operation of changing $D^{\prime}$ into $D^{\prime \prime}$, if there exists a diagonal matrix $S$ with diagonal entries in $\{ \pm 1, \pm \mathrm{i}\}$ such that $H\left(D^{\prime \prime}\right)=S^{-1} H\left(D^{\prime}\right) S$.

Proposition 4. $[8,13]$ The four-way switching on any mixed graph D gives a mixed graph that is cospectral with $D$.

By Definition 3, it is easy to see that if $D^{\prime \prime}$ is obtained from $D^{\prime}$ by a sequence of four-way switchings, then $D^{\prime \prime}$ can be obtained from $D^{\prime}$ by a single four-way switching. In $[8,13]$, the authors described the four-way switching as a special transformation between two mixed graphs with the same underlying graph. Their work implies that if $D^{\prime}$ and $D^{\prime \prime}$ are two mixed forests with the same underlying graph, then $D^{\prime \prime}$ can be obtained from $D^{\prime}$ by a four-way switching. This is naturally generalized as follows.

Lemma 5. Let $D^{\prime}$ and $D^{\prime \prime}$ be two connected mixed graphs with the same underlying graph of order $n$. Then $D^{\prime \prime}$ can be obtained from $D^{\prime}$ by a four-way switching if and only if all corresponding cycles of $D^{\prime}$ and $D^{\prime \prime}$ have the same values.

Proof. Let $H\left(D^{\prime}\right)=\left[h_{u v}^{\prime}\right]$ and $H\left(D^{\prime \prime}\right)=\left[h_{u v}^{\prime \prime}\right]$.

Necessity. By Definition 3, there is a diagonal matrix $S=\operatorname{diag}\left\{s_{1}, \ldots, s_{n}\right\}$ such that

$$
h_{u v}^{\prime \prime}=s_{u}^{-1} h_{u v}^{\prime} s_{v}
$$

for any $u v \in E\left(D^{\prime}\right)$. Let $C^{\prime}=v_{1} v_{2} \ldots v_{l} v_{1}$ be a cycle of $D^{\prime}$. Denote the corresponding cycle of $C^{\prime}$ in $D^{\prime \prime}$ by $C^{\prime \prime}$. We have

$$
\begin{aligned}
h\left(C^{\prime \prime}\right) & =h_{v_{1} v_{2}}^{\prime \prime} h_{v_{2} v_{3}}^{\prime \prime} \cdots h_{v_{l} v_{1}}^{\prime \prime} \\
& =s_{v_{1}}^{-1} h_{v_{1} v_{2}}^{\prime} s_{v_{2}} s_{v_{2}}^{-1} h_{v_{2} v_{3}}^{\prime} s_{v_{3}} \cdots s_{v_{l}}^{-1} h_{v_{l} v_{1}}^{\prime} s_{v_{1}} \\
& =h_{v_{1} v_{2}}^{\prime} h_{v_{2} v_{3}}^{\prime} \cdots h_{v_{l} v_{1}}^{\prime}=h\left(C^{\prime}\right) .
\end{aligned}
$$

We prove the sufficiency by induction on $k$, the dimension of the cycle space of $D^{\prime}$. If $k=0$, then $D^{\prime}$ and $D^{\prime \prime}$ are two mixed trees with the same underlying graph, and thus the conclusion follows.

Assume that $k \geqslant 1$ and that the assertion holds for the mixed graphs with dimension less than $k$. Let $D^{\prime}$ be a mixed graph with dimension $k$. Take a cycle $C^{\prime}=v_{1} v_{2} \ldots v_{l} v_{1}$ in $D^{\prime}$ and an edge $e^{\prime}=v_{1} v_{2} \in E\left(C^{\prime}\right)$. The corresponding cycle and edge in $D^{\prime \prime}$ is denoted by $C^{\prime \prime}$ and $e^{\prime \prime}$, respectively. By the induction hypothesis, $D^{\prime \prime}-e^{\prime \prime}$ can be obtained from $D^{\prime}-e^{\prime}$ by a four-way switching, that is, there is a diagonal matrix $S=\operatorname{diag}\left\{s_{1}, \ldots, s_{n}\right\}$ such that $h_{u v}^{\prime \prime}=s_{u}^{-1} h_{u v}^{\prime} s_{v}$ for any edge $u v \in E\left(D^{\prime}-e^{\prime}\right)$. By Definition 3, it is enough to prove $h_{v_{1} v_{2}}^{\prime \prime}=s_{v_{1}}^{-1} h_{v_{1} v_{2}}^{\prime} s_{v_{2}}$. Note that $h\left(C^{\prime \prime}\right)=h\left(C^{\prime}\right)$, that is,

$$
h_{v_{1} v_{2}}^{\prime \prime} h_{v_{2} v_{3}}^{\prime \prime} \cdots h_{v_{l} v_{1}}^{\prime \prime}=h_{v_{1} v_{2}}^{\prime} h_{v_{2} v_{3}}^{\prime} \cdots h_{v_{l} v_{1}}^{\prime} \text {. }
$$

It implies that $h_{v_{1} v_{2}}^{\prime \prime}=s_{v_{1}}^{-1} h_{v_{1} v_{2}}^{\prime} s_{v_{2}}$. 
A mixed graph is positive if each cycle is positive. By Lemma 5, we immediately have the following corollary which was given by Liu and Li in [11].

Corollary 6. [11] Let $D$ be a mixed graph with the underlying graph $G$. Then $D$ is positive if and only if $D$ can be obtained from $G$ by a four-way switching.

Reversing all directed edges in mixed graph $D$, we obtain a mixed graph, called the converse of $D$, denoted by $D^{\top}$. Obviously, $D^{\top}$ is cospectral to $D$ because of $H\left(D^{\top}\right)=$ $H(D)^{\top}$. We immediately obtain the following lemma.

Lemma 7. Let $D$ be a mixed graph, and let $C$ and $C^{\prime}$ be two corresponding cycles in $D$ and $D^{\top}$, respectively. Then $h(C)=\overline{h\left(C^{\prime}\right)}$.

By Lemma 7, the converse operation is different from the four-way switching operation because it does not preserve the cycle values while the four-way switching does. Mohar [13] defined that two mixed graphs $D_{1}$ and $D_{2}$ are switching equivalent if one can be obtained from the other by a sequence of four-way switchings and converse operations.

Proposition 8. [13] For each graph $G$, switching equivalence gives an equivalence relation on $\mathcal{D}(G)$.

Clearly, cospectral relation is an equivalence relation on $\mathcal{D}(G)$. Denote by $n(G)$ the number of cospectral equivalence classes of $\mathcal{D}(G)$. By Proposition 4, it is clear that $n(G) \leqslant n_{s}(G)$.

The proof of Proposition 8 (Proposition 3.3 in [13]) implies the following lemma.

Lemma 9. [13] Let $D^{\prime}$ and $D^{\prime \prime}$ be two mixed graphs with the same underlying graph. If $D^{\prime \prime}$ can be obtained from $D^{\prime}$ by a four-way switching and then taking the converse, then $D^{\prime \prime}$ can be obtained by a four-way switching from the converse of $D^{\prime}$.

By Lemma 7, Proposition 8 and Lemma 9, the definition of switching equivalence can be reduced as follows.

Definition 10. [13] Two mixed graphs $D^{\prime}$ and $D^{\prime \prime}$ are switching equivalent if one can be obtained from the other by a four-way switching and taking a possible converse.

By Lemma 5, Lemma 7 and Definition 10, we present the main theorem of this section, which characterizes the switching equivalence between two mixed graphs.

Theorem 11. Let $D^{\prime}$ and $D^{\prime \prime}$ be two connected mixed graphs with the same underlying graph. Then $D^{\prime}$ is switching equivalent to $D^{\prime \prime}$ if and only if all corresponding cycles of $D^{\prime}$ and $D^{\prime \prime}$ have the same values or the conjugate values.

Usually, it is difficult to find all corresponding cycles of $D^{\prime}$ and $D^{\prime \prime}$ and compare their values while the edge density is enough large. Thus, Theorem 11 is only a rough characterization on switching equivalence. To refine it, the strong cycle basis, a special cycle basis, will be introduced in next section. 


\section{Strong cycle basis}

The graphs considered in this section maybe have multiple edges, but have no loops. Denote by $\mathcal{E}(G)$ the set of all subsets of $E(G)$ for a graph $G$. This set forms a vector space over the 2-element field $\mathbb{F}_{2}$ under the symmetric difference, which is called the edge space of $G$. The cycle space $\mathcal{C}(G)$ is the subspace of $\mathcal{E}(G)$ spanned by the edge sets of cycles in $G$. In the other word, $\mathcal{C}(G)$ can be considered as the subspace of $\mathcal{E}(G)$ consisting of edge sets of all even subgraphs (that is, the subgraph whose all vertices have even degree) in $G$. Accordingly, denote by $\tilde{\mathcal{C}}(G)$ the set consisting of all cycles in $G$.

The dimension of the cycle space is equal to $|E(G)|-|V(G)|+s(G)$, where $s(G)$ is the number of connected components of $G$. A connected graph is said to be $k$-cyclic if the dimension of its cycle space is $k$. A cycle basis of $G$ is a basis for the cycle space of $G$ which consists entirely of cycles. Let $\mathscr{C}=\left\{E\left(C_{1}\right), \ldots, E\left(C_{k}\right)\right\}$ be a cycle basis of $G$. For convenience, we write $\left\{C_{1}, \ldots, C_{k}\right\}$ instead of $\left\{E\left(C_{1}\right), \ldots, E\left(C_{k}\right)\right\}$. It is known that all induced cycles can generate cycle space which implies the existence of cycle basis for any graph.

Let $G_{1}$ and $G_{2}$ be two subgraphs of $G$. The symmetric difference of $G_{1}$ and $G_{2}$, denoted by $G_{1} \oplus G_{2}$, is defined to be a subgraph of $G$ induced by the symmetric difference of $E\left(G_{1}\right)$ and $E\left(G_{2}\right)$. Notably, it is possible that the symmetric difference of two connected graphs is not connected.

Let $C_{1}$ and $C_{2}$ be two cycles of $G$. It is easy to see that $C_{1} \oplus C_{2}$ is an even graph. If the intersection of $C_{1}$ and $C_{2}$ is exactly a non-trivial path $P_{k}(k \geqslant 2)$, we say that cycle $C_{1} \oplus C_{2}$ is obtained from $C_{1}$ and $C_{2}$ by making a strong symmetric difference, and write $C_{1} \oplus_{s} C_{2}$ instead of $C_{1} \oplus C_{2}$.

Let $\mathscr{F}$ be a family of cycles of $D$. We recursively construct the family $\widehat{\mathscr{F}}$ as follows:

(1) Initially, $\widehat{\mathscr{F}}=\mathscr{F}$.

(2) For any cycle $C \in \tilde{\mathcal{C}}(G)$, if there are two cycles $C_{1}$ and $C_{2}$ in $\widehat{\mathscr{F}}$ such that $C=C_{1} \oplus_{s} C_{2}$, then we add $C$ to $\widehat{\mathscr{F}}$.

The above process will terminate as $G$ is a finite graph and we finally obtain a set $\widehat{\mathscr{F}}$, which is called the closed set of $\mathscr{F}$ with respect to strong symmetric difference.

Definition 12. Let $G$ be a connected graph. A set $\mathscr{C} \subseteq \tilde{\mathcal{C}}(G)$ is called a strong cycle basis of $G$ if

(1) $\mathscr{C}$ is a cycle basis of $G$;

(2) $\widehat{\mathscr{C}}=\tilde{\mathcal{C}}(G)$.

Remark 13. To show $\mathscr{C}=\left\{C_{1}, \ldots, C_{k}\right\}$ being a strong cycle basis of $k$-cyclic graph $G$, it is sufficient to verify that $\hat{\mathscr{C}}=\tilde{\mathcal{C}}(G)$.

The existence of cycle basis with some constraints is an important topic of cycle theory, and has received wide attention. One can refer to $[1,15]$ and references therein for more details. In the following, we present the main theorem in this section.

Theorem 14. Every graph admits a strong cycle basis. 


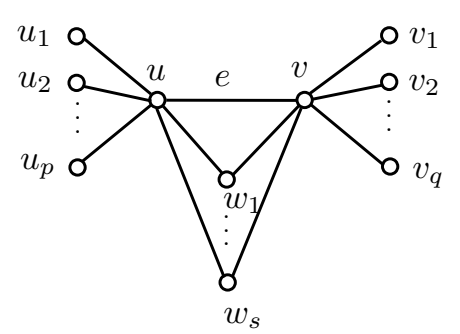

$G$

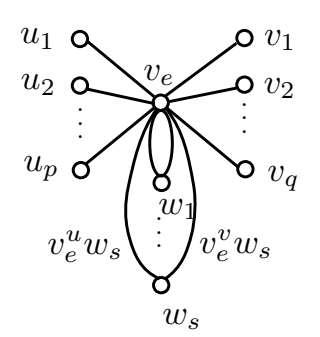

$G / e$

Figure 1: $G$ and $G / e$.

Proof. Without loss of generality, we may assume that the graph considered is connected. Otherwise, the cycle space is the direct sum of the cycle spaces of the connected components.

Let $G$ be a counterexample with the minimum number of edges. We can claim that $G$ is a connected $k$-cyclic graph for $k \geqslant 2$, since the assertion is trivial for trees and unicyclic graphs.

We may claim that $G$ is 2-connected. If not, let $v$ be a cut vertex of $G$. Then, $G$ can be obtained from a number of connected subgraphs $G_{1}, \ldots, G_{l}(l \geqslant 2)$ of $G$ by identifying some vertex in each $G_{i}$ at a common vertex $v$. Since $\left|E\left(G_{i}\right)\right|<|E(G)|$ for each $i(1 \leqslant i \leqslant l), G_{i}$ contains a strong cycle basis, say $\mathscr{C}_{i}$. It is easy to verify that $\mathscr{C}_{1} \cup \cdots \cup \mathscr{C}_{l}$ is a strong cycle basis of $G$.

Take an edge $e=u v$ in $G$. As $G$ is 2-connected, $e$ is not a cut edge. We consider the following cases.

Case 1: There is another edge $f$ joining $u$ and $v$.

Since $G-e$ is a connected $(k-1)$-cyclic graph, $G-e$ admits a strong cycle basis, say $\left\{C_{1}, \ldots, C_{k-1}\right\}$. Denote by $C_{0}$ the digon consisting of $e$ and $f$. We prove that $\mathscr{C}:=$ $\left\{C_{0}, C_{1}, \ldots, C_{k-1}\right\}$ is a strong cycle basis of $G$, which contradicts to the choice of $G$. By Remark 13, it is enough to prove that $C \in \widehat{\mathscr{C}}$ for any cycle $C \in \tilde{\mathcal{C}}(G) \backslash \mathscr{C}$. If $e \notin E(C)$, then $C \in \hat{\mathscr{C}}$ as $C \in \widehat{\mathscr{C}}(G-e)$. If $e \in E(C)$, replacing $e$ by $f$, we can obtain a cycle in $G-e$, denoted by $C^{\prime}$. Note that $C=C^{\prime} \oplus_{s} C_{0}$ and $C^{\prime} \in \widehat{\mathscr{C}}(G-e) \subset \widehat{\mathscr{C}}$. So, $C \in \widehat{\mathscr{C}}$.

Case 2: The edge $e$ is a unique edge joining $u$ and $v$.

Let $U=\left\{u_{1}, \ldots, u_{p}\right\}$ be the set of vertices which are adjacent to $u$, but not to $v$. Let $V=\left\{v_{1}, \ldots, v_{q}\right\}$ be the set of vertices which are adjacent to $v$, but not to $u$. Let $W=\left\{w_{1}, \ldots, w_{s}\right\}$ be the set of vertices which are adjacent to both $u$ and $v$. Denote by $G / e$ the graph obtained from $G$ by contracting the edge $e$ into a new vertex $v_{e}$, which becomes adjacent to all the former neighbors of $u$ and $v$. In particular, the edge $u w_{i}$ and $v w_{i}$ are relabeled as two parallel edges between $v_{e}$ and $w_{i}$ for $1 \leqslant i \leqslant s$, denoted by $v_{e}^{u} w_{i}$ and $v_{e}^{v} w_{i}$, respectively, see Figure 1 .

Now, we define a mapping $\phi$ from $\tilde{\mathcal{C}}(G / e)$ to $\tilde{\mathcal{C}}(G)$ as follows, which is the key of remaining proof. For any $C \in \tilde{\mathcal{C}}(G / e)$, if $v_{e} \notin C$, then $\phi(C)$ is the cycle corresponding to 

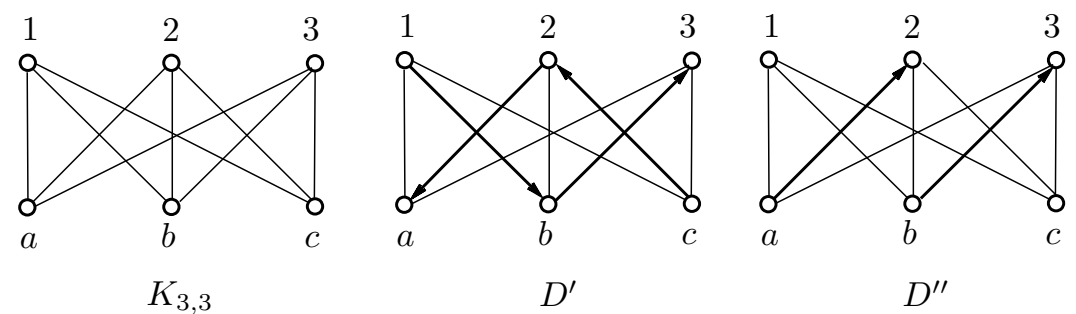

Figure $2: K_{3,3}$ and its two orientations.

$C$ in $G$. If $v_{e} \in C$, we denote $f_{1} v_{e} f_{2}$ to be the segment of $C$ containing $v_{e}$. Let

$$
\begin{aligned}
& E_{u}=\left\{v_{e} u_{1}, \ldots, v_{e} u_{p}, v_{e}^{u} w_{1}, \ldots, v_{e}^{u} w_{s}\right\}, \\
& E_{v}=\left\{v_{e} v_{1}, \ldots, v_{e} v_{q}, v_{e}^{v} w_{1}, \ldots, v_{e}^{v} w_{s}\right\} .
\end{aligned}
$$

Since $e$ is the unique edge between $u$ and $v$, contracting $e$ from $G$ does not create loops. Then, $E_{u} \cup E_{v}$ is the set consisting of all edges incident to $v_{e}$ in $G / e$. If $f_{1}$ and $f_{2}$ lie in the same set, say $E_{u}$, then $\phi(C)$ is defined to be the cycle in $G$ obtained from $C$ by replacing the segment $f_{1} v_{e} f_{2}$ by the segment of $f_{1}^{\prime} u f_{2}^{\prime}$, where $f_{i}^{\prime}$ is the edge in $G$ corresponding to $f_{i}$ for $i=1,2$. If $f_{1} \in E_{u}, f_{2} \in E_{v}$, then $\phi(C)$ is defined to be the cycle in $G$ obtained from $C$ by replacing the segment $f_{1} v_{e} f_{2}$ by the segment of $f_{1}^{\prime} u e v f_{2}^{\prime}$.

Let $C, C^{\prime}, C^{\prime \prime}$ be three cycles in $G / e$. It is clear that if $C=C^{\prime} \oplus_{s} C^{\prime \prime}$, then $\phi(C)=$ $\phi\left(C^{\prime}\right) \oplus_{s} \phi\left(C^{\prime \prime}\right)$. Since $G / e$ is a connected $k$-cyclic graph with fewer number of edges and without loops, $G / e$ admits a strong cycle basis, say $\left\{C_{1}, \ldots, C_{k}\right\}$. Next, we prove that $\phi(\mathscr{C})=\left\{\phi\left(C_{1}\right), \ldots, \phi\left(C_{k}\right)\right\}$ is a strong cycle basis of $G$, which yields a contradiction.

For any cycle $\tilde{C}$ in $G$, if there exists a cycle $C$ in $G / e$ such that $\phi(C)=\tilde{C}$, it is easy to check that $\tilde{C} \in \widehat{\phi(\mathscr{C})}$. If not, it implies that $u, v \in \tilde{C}$ and $e \notin \tilde{C}$. We rewrite $\tilde{C}$ by $u P_{1} v P_{2} u$, where $P_{1}$ and $P_{2}$ are paths with length at least two. Denote by $\tilde{C}^{\prime}\left(\tilde{C}^{\prime \prime}\right.$, resp.) the cycle obtained from $\tilde{C}$ by replacing $P_{1}\left(P_{2}\right.$, resp.) by $e$. By the above discussion, $\tilde{C}^{\prime} \in \widehat{\phi(\mathscr{C})}$ and $\tilde{C}^{\prime \prime} \in \widehat{\phi(\mathscr{C})}$. Since $\tilde{C}=\tilde{C}^{\prime} \oplus_{s} \tilde{C}^{\prime \prime}$, we have $\tilde{C} \in \widehat{\phi(\mathscr{C})}$.

Remark 15. Not all cycle bases are strong cycle bases. Consider the complete bipartite graph $K_{3,3}$ with vertex labeling shown in Figure 2. Let $C_{1}=1 b 3 c 2 a 1, C_{2}=1 b 2 c 3 a 1$, $C_{3}=1 c 2 b 3 a 1$ and $C_{4}=1 c 3 b 2 a 1$. It is easy to check that $\mathscr{C}=\left\{C_{1}, C_{2}, C_{3}, C_{4}\right\}$ is a cycle basis of $K_{3,3}$, but not a strong cycle basis, since $\widehat{\mathscr{C}}=\mathscr{C}$.

\section{The number of switching equivalence classes}

Based primarily on strong cycle basis, in this section, we will refine the characterization of switching equivalence between two mixed graphs in Section 2, and give a method to estimate the number of switching equivalence classes in $\mathcal{D}(G)$. Using this method, an upper bound for $n_{s}(G)$ is obtained. 


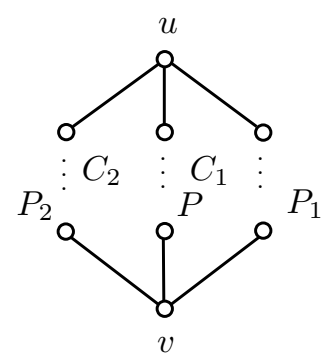

Figure 3: Strong symmetric difference of two cycles.

Lemma 16. Let $C, C_{1}, C_{2}$ be three cycles of simple graph $G$ satisfying $C=C_{1} \oplus_{s} C_{2}$, and let $w_{1}, w_{2} \in\{ \pm 1, \pm \mathbf{i}\}$ be two complex numbers. Under any given cycle orientation of $G$, $h(C)$ is determined by $h\left(C_{1}\right)$ and $h\left(C_{2}\right)$. That is, for any mixed graph $D \in \mathcal{D}(G)$ with $h\left(C_{1}\right)=w_{1}$ and $h\left(C_{2}\right)=w_{2}$, the value of $C$ only depends on $w_{1}$ and $w_{2}$.

Proof. Let $C_{1}=u P v P_{1} u, C_{2}=u P_{2} v P u$, and $C=u P_{2} v P_{1} u$ (see Figure 3). Without loss of generality, we calculate the values of $C, C_{1}$ and $C_{2}$ anticlockwise, considering the values of $P, P_{1}$ and $P_{2}$ in the direction from $u$ to $v$. For any mixed graph $D \in \mathcal{D}(G)$ with $h\left(C_{1}\right)=w_{1}$ and $h\left(C_{2}\right)=w_{2}$, we have

$$
h(C)=\overline{h\left(P_{1}\right)} h\left(P_{2}\right)=\overline{h\left(P_{1}\right)} h(P) \overline{h(P)} h\left(P_{2}\right)=h\left(C_{1}\right) h\left(C_{2}\right)=w_{1} w_{2} .
$$

The result follows.

Remark 17. In Lemma 16, if $C=C_{1} \oplus C_{2}$, then $h(C)$ may be not determined by $h\left(C_{1}\right)$ and $h\left(C_{2}\right)$. For example, consider two mixed graphs $D^{\prime}$ and $D^{\prime \prime}$ shown in Figure 2. Let $C_{1}=1 b 3 c 2 a 1, C_{2}=1 b 2 c 3 a 1$ and $C=a 2 b 3 a$. Then $C=C_{1} \oplus C_{2}$ and $C \neq C_{1} \oplus_{s} C_{2}$. In $D^{\prime}, h\left(C_{1}\right)=h\left(C_{2}\right)=h(C)=1$, however, in $D^{\prime \prime}, h\left(C_{1}\right)=h\left(C_{2}\right)=1$ and $h(C)=-1$.

In the proof of Lemma 16, if the directions of $C$ and $C_{1}$ are anticlockwise, the direction of $C_{2}$ is clockwise, it follows from the proof that $h(C)=h\left(C_{1}\right) \overline{h\left(C_{2}\right)}$. Hereafter, the directions of $C_{1}, C_{2}$ and $C$ are said to be coincident if $h(C)=h\left(C_{1}\right) h\left(C_{2}\right)$ under these cycle orientations.

Let $G$ be a connected $k$-cyclic simple graph. By Theorem 14, $G$ admits a strong cycle basis, denoted by $\mathscr{C}=\left\{C_{1}, \ldots, C_{k}\right\}$. For any mixed graph $D \in \mathcal{D}(G)$ and its given cycle orientation, if we fix the values of $C_{1}, \ldots, C_{k}$ in $D$, by Lemma 16 , the values of all cycles in $\tilde{\mathcal{C}}(D)$ are determined. Based on this, ordered sequence $\mathfrak{s}(D)=\left(h\left(C_{1}\right), \ldots, h\left(C_{k}\right)\right)$ is called an essential vector of $D$, which depends on $\mathscr{C}$ and the given cycle orientation.

Let $D^{\prime}$ and $D^{\prime \prime}$ be two mixed graphs with the same underlying graph $G$. We always assume that $D^{\prime}$ and $D^{\prime \prime}$ have the same cycle orientation. Let $\mathfrak{s}\left(D^{\prime}\right)=\left(h\left(C_{1}^{\prime}\right), \ldots, h\left(C_{k}^{\prime}\right)\right)$ and $\mathfrak{s}\left(D^{\prime \prime}\right)=\left(h\left(C_{1}^{\prime \prime}\right), h\left(C_{2}^{\prime \prime}\right), \ldots, h\left(C_{k}^{\prime \prime}\right)\right)$, where $C_{j}^{\prime}\left(C_{j}^{\prime \prime}\right.$, resp. $)$ is the corresponding cycle in $D^{\prime}\left(D^{\prime \prime}\right.$, resp.) of $C_{j}$ for any $j(1 \leqslant j \leqslant k)$. For any cycle $C^{\prime}$ of $D^{\prime}$ and its corresponding cycle $C^{\prime \prime}$ of $D^{\prime \prime}$, it is easy to see that $h\left(C^{\prime}\right)=h\left(C^{\prime \prime}\right)$ if $\mathfrak{s}\left(D^{\prime}\right)=\mathfrak{s}\left(D^{\prime \prime}\right) ; h\left(C^{\prime}\right)=\overline{h\left(C^{\prime \prime}\right)}$ if $\mathfrak{s}\left(D^{\prime}\right)=\overline{\mathfrak{s}\left(D^{\prime \prime}\right)}$. Two vectors $\mathfrak{s}\left(D^{\prime}\right)$ and $\mathfrak{s}\left(D^{\prime \prime}\right)$ are said to be conjugate equivalent if 
$\mathfrak{s}\left(D^{\prime}\right)=\mathfrak{s}\left(D^{\prime \prime}\right)$ or $\mathfrak{s}\left(D^{\prime}\right)=\overline{\mathfrak{s}\left(D^{\prime \prime}\right)}$. Using this terminology, we refine Theorem 11 as follows.

Theorem 18. Let $D^{\prime}$ and $D^{\prime \prime}$ be two mixed graphs with the same underlying graph. Two mixed graphs $D^{\prime}$ and $D^{\prime \prime}$ are switching equivalent if and only if $\mathfrak{s}\left(D^{\prime}\right)$ and $\mathfrak{s}\left(D^{\prime \prime}\right)$ are conjugate equivalent for any fixed strong cycle basis and cycle orientation.

Proof. It follows from Theorem 11 and Lemma 16.

Denote by $\mathcal{S}(G)$ the set of essential vectors of all mixed graphs in $\mathcal{D}(G)$. The conjugate equivalence naturally defines an equivalence relation on $\mathcal{S}(G)$. By Theorem 18, the determination of $n_{s}(G)$ is equivalent to find the number of conjugate equivalence classes in $\mathcal{S}(G)$. As an application, a natural upper bound of $n_{s}(G)$ is given in following theorem.

Theorem 19. For any connected $k$-cyclic graph $G, n_{s}(G) \leqslant 2^{2 k-1}+2^{k-1}$.

Proof. Let $x_{j}$ be the number of conjugate equivalence classes in $\mathcal{S}(G)$ whose essential vectors have exactly $j$ imaginary entries at the same position. For $j=0$, the corresponding essential vectors are real, so $x_{0} \leqslant 2^{k}$. For $1 \leqslant j \leqslant k, x_{j} \leqslant \frac{1}{2}\left(\begin{array}{c}k \\ j\end{array}\right) 2^{k}$. Therefore

$$
n_{s}(G)=\sum_{j=0}^{k} x_{j} \leqslant 2^{k}+\sum_{j=1}^{k} \frac{1}{2}\left(\begin{array}{l}
k \\
j
\end{array}\right) 2^{k}=2^{2 k-1}+2^{k-1} .
$$

This completes the proof.

A connected $k$-cyclic graph $G$ is called free with respect to a strong cycle basis $\mathscr{C}$ if for any vector $s=\left\{s_{1}, \ldots, s_{k}\right\}$ whose components are in $\{ \pm 1, \pm \mathrm{i}\}$, there exists $D \in \mathcal{D}(G)$ such that $\mathfrak{s}(D)=s$. Clearly, the equality in Theorem 19 holds if and only if $G$ is free with respect to some strong cycle basis. Unfortunately, not all graphs are free. While the edge density of a graph is enough large, it may be not free. For instance, it follows from a rough estimation that $K_{n}$ is not free for $n \geqslant 11$. Here, we only give an obvious lemma which will be used in Section 5 .

Lemma 20. Let $\mathscr{C}=\left\{C_{1}, \ldots, C_{k}\right\}$ be a strong cycle basis of $G$. If for any $i(2 \leqslant i \leqslant k)$, $C_{i}$ contains at least two edges which are not in other cycles in $\mathscr{C}$, then $G$ is free with respect to $\mathscr{C}$.

\section{ODHS graphs}

We first introduce some families of graphs to be used later. Let $G$ be a connected $k$-cyclic graph. The kernel of $G$, denoted by $\hat{G}$, is the unique minimal connected induced $k$-cyclic subgraph of $G$. It is clear that $\hat{G}$ is the unique $k$-cyclic induced subgraph of $G$ containing no pendant vertices, and $G$ can be obtained from $\hat{G}$ by attaching trees to some vertices of $\hat{G}$. A 2-cyclic graph is also called a bicyclic graph. A theta graph, denoted by $\theta\left(l_{1}, l_{2}, l_{3}\right)$, is a bicyclic graph consisting of three internally disjoint paths $P_{1}, P_{2}, P_{3}$ with the same 
endpoints, where $P_{i}$ has length $l_{i}$ for each $i$. A bicyclic graph $G$ is said to be of theta-type graph, if $\hat{G}$ is a theta graph. Hereafter, we always assume that $l_{1} \leqslant l_{2} \leqslant l_{3}$, and denote $C_{i j}$ to be the cycle consisting of two paths $P_{i}$ and $P_{j}$ for $1 \leqslant i<j \leqslant 3$.

Let $D^{\prime}$ and $D^{\prime \prime}$ be two mixed graphs with the same underlying graph $G$. If $C$ is a cycle of $G$, we always denote the corresponding cycle of $C$ in $D^{\prime}$ ( $D^{\prime \prime}$, resp.) by $C^{\prime}\left(C^{\prime \prime}\right.$, resp.).

In this section, we will characterize all bicyclic ODHS graphs, and construct infinitely many $k$-cyclic ODHS graphs, both of which can achieve the upper bound in Theorem 19. Note that two switching equivalent mixed graphs are cospectral. By the definition of ODHS graph, we immediately have the following lemma.

Lemma 21. A simple graph $G$ is $O D H S$ if and only if $n(G)=n_{s}(G)$.

By Theorem 11, all mixed trees with the same underlying graph are switching equivalent, which implies that all trees are ODHS. For unicyclic graph $G$, by Theorem 1 and Theorem 11, it is clear that $n(G)=n_{s}(G)=3$, which implies that all unicyclic graphs are ODHS. Thus, it is natural to ask which $k$-cyclic graphs are ODHS for $k \geqslant 2$. To answer this question, the following lemmas are needed.

Lemma 22. Let $D^{\prime}$ and $D^{\prime \prime}$ be two mixed graphs with underlying graph $G$, and let $\Phi\left(D^{\prime}, \lambda\right)=\sum_{j=0}^{n} c_{j}^{\prime} \lambda^{n-j}\left(\Phi\left(D^{\prime \prime}, \lambda\right)=\sum_{j=0}^{n} c_{j}^{\prime \prime} \lambda^{n-j}\right.$, resp. $)$ be the characteristic polynomial of $D^{\prime}\left(D^{\prime \prime}\right.$, resp.). If the corresponding basic cycles whose lengths are less than $p$ have the same values, then $c_{j}^{\prime}=c_{j}^{\prime \prime}$ for any $j(1 \leqslant j \leqslant p-1)$. Furthermore, if the difference of the number of positive cycles and the number of negative cycles of order $p$ in $D^{\prime}$ is not equal to that in $D^{\prime \prime}$, then $c_{p}^{\prime} \neq c_{p}^{\prime \prime}$.

Proof. Let $E_{j}^{\prime}$ and $E_{j}^{\prime \prime}$ be two basic subgraphs of order $j$ containing at least one cycle of $D^{\prime}$ and $D^{\prime \prime}$, respectively. Let $M_{j}^{\prime}$ and $M_{j}^{\prime \prime}$ be two basic subgraphs of order $j$ (for even $j$ ) consisting of $j / 2$ isolated edges of $D^{\prime}$ and $D^{\prime \prime}$, respectively. By Theorem 1 , for any $j(1 \leqslant j \leqslant p)$,

$$
\begin{aligned}
c_{j}^{\prime}-c_{j}^{\prime \prime}= & \sum_{B_{j}^{\prime}} \prod_{C \subset B_{j}^{\prime}} h(C)(-1)^{s\left(B_{j}^{\prime}\right)} 2^{c\left(B_{j}^{\prime}\right)}-\sum_{B_{j}^{\prime \prime}} \prod_{C \subset B_{j}^{\prime \prime}} h(C)(-1)^{s\left(B_{j}^{\prime \prime}\right)} 2^{c\left(B_{j}^{\prime \prime}\right)} \\
= & \left(\sum_{E_{j}^{\prime}} \prod_{C \subset E_{j}^{\prime}} h(C)(-1)^{s\left(E_{j}^{\prime}\right)} 2^{c\left(E_{j}^{\prime}\right)}+\sum_{M_{j}^{\prime}}(-1)^{s\left(M_{j}^{\prime}\right)}\right) \\
& -\left(\sum_{E_{j}^{\prime \prime}} \prod_{C \subset E_{j}^{\prime \prime}} h(C)(-1)^{s\left(E_{j}^{\prime \prime}\right)} 2^{c\left(E_{j}^{\prime \prime}\right)}+\sum_{M_{j}^{\prime \prime}}(-1)^{s\left(M_{j}^{\prime \prime}\right)}\right) \\
= & \sum_{E_{j}^{\prime}} \prod_{C \subset E_{j}^{\prime}} h(C)(-1)^{s\left(E_{j}^{\prime}\right)} 2^{c\left(E_{j}^{\prime}\right)}-\sum_{E_{j}^{\prime \prime}} \prod_{C \subset E_{j}^{\prime \prime}} h(C)(-1)^{s\left(E_{j}^{\prime \prime}\right)} 2^{c\left(E_{j}^{\prime \prime}\right)} .
\end{aligned}
$$

Note that for corresponding basic subgraphs $E_{j}^{\prime}$ and $E_{j}^{\prime \prime}, s\left(E_{j}^{\prime}\right)=s\left(E_{j}^{\prime \prime}\right)$ and $c\left(E_{j}^{\prime}\right)=c\left(E_{j}^{\prime \prime}\right)$. It is easy to check that $c_{j}^{\prime}=c_{j}^{\prime \prime}$ for any $j(1 \leqslant j \leqslant p-1)$ and $c_{p}^{\prime} \neq c_{p}^{\prime \prime}$. The result follows. 
Lemma 23. Let $G$ be a connected simple graph. If there exists a cycle $C$ of $G$ which has no common edge with other cycles of $G$, then $G$ is not ODHS.

Proof. By Lemma 21, it is sufficient to prove that $n(G)<n_{s}(G)$. Since $C$ is edgedisjoint to the other cycles, $C$ must be contained in any strong cycle basis of $G$. Let $\left\{C, C_{1}, \ldots, C_{k-1}\right\}$ be a strong cycle basis of $G$. We will complete the proof by constructing two mixed graphs $D^{\prime}$ and $D^{\prime \prime}$ in $\mathcal{D}(G)$ which are cospectral but not switching equivalent. Noting that the direction of $E(C)$ is independent of the directions of the rest cycles, we construct two mixed graphs $D^{\prime}$ and $D^{\prime \prime}$ in $\mathcal{D}(G)$ satisfying:

(1) $h\left(C^{\prime}\right)=\mathrm{i}, h\left(C^{\prime \prime}\right)=-\mathrm{i}$;

(2) $\left(h\left(C_{1}^{\prime}\right), \ldots, h\left(C_{k-1}^{\prime}\right)\right)=\left(h\left(C_{1}^{\prime \prime}\right), \ldots, h\left(C_{k-1}^{\prime \prime}\right)\right)$ is not a real vector.

Clearly, $\mathfrak{s}\left(D^{\prime}\right)$ and $\mathfrak{s}\left(D^{\prime \prime}\right)$ are not conjugate equivalent. It follows from Theorem 18 that $D^{\prime}$ and $D^{\prime \prime}$ are not switching equivalent. Since $C^{\prime}\left(C^{\prime \prime}\right.$, resp.) is imaginary and edge-disjoint to the other cycles in $D^{\prime}$ ( $D^{\prime \prime}$, resp.), any basic cycle in $D^{\prime}$ ( $D^{\prime \prime}$, resp.) can be generated by $\left\{C_{1}^{\prime}, \ldots, C_{k-1}^{\prime}\right\}\left(\left\{C_{1}^{\prime \prime}, \ldots, C_{k-1}^{\prime \prime}\right\}\right.$, resp.). By Lemma 16 and the fact $\left(h\left(C_{1}^{\prime}\right), \ldots, h\left(C_{k-1}^{\prime}\right)\right)=\left(h\left(C_{1}^{\prime \prime}\right), \ldots, h\left(C_{k-1}^{\prime \prime}\right)\right)$, one can deduce that all corresponding basic cycles in $D^{\prime}$ and $D^{\prime \prime}$ have the same value. By Lemma $22, c_{j}^{\prime}=c_{j}^{\prime \prime}$ for any $j$, which implies that $\Phi\left(D^{\prime}, \lambda\right)=\Phi\left(D^{\prime \prime}, \lambda\right)$.

In what follows, we characterize bicyclic ODHS graphs. By Lemma 23, the question can be reduced to determine which theta-type graphs are ODHS.

Lemma 24. Let $G$ be a theta-type bicyclic graph and let $D^{\prime}$ and $D^{\prime \prime}$ be two mixed graphs with underlying graph $G$. Then $D^{\prime}$ is cospectral to $D^{\prime \prime}$ if and only if

$$
\sum_{C^{\prime} \in \mathcal{C}_{v}^{\prime}} h\left(C^{\prime}\right) \Phi\left(D^{\prime}-C^{\prime}, \lambda\right)=\sum_{C^{\prime \prime} \in \mathcal{C}_{v}^{\prime \prime}} h\left(C^{\prime \prime}\right) \Phi\left(D^{\prime \prime}-C^{\prime \prime}, \lambda\right)
$$

where $v$ is one of the common vertices of all cycles in $G$, and $\mathcal{C}_{v}^{\prime}\left(\mathcal{C}_{v}^{\prime \prime}\right.$, resp.) is the set of basic cycles in $D^{\prime}$ ( $D^{\prime \prime}$, resp.) containing $v$.

Proof. By Theorem 2, we have

$$
\Phi\left(D^{\prime}, \lambda\right)=\lambda \Phi\left(D^{\prime}-v, \lambda\right)-\sum_{u v \in E\left(D^{\prime}\right)} \Phi\left(D^{\prime}-u-v, \lambda\right)-2 \sum_{C^{\prime} \in \mathcal{C}_{v}^{\prime}} h\left(C^{\prime}\right) \Phi\left(D^{\prime}-C^{\prime}, \lambda\right)
$$

and

$$
\Phi\left(D^{\prime \prime}, \lambda\right)=\lambda \Phi\left(D^{\prime \prime}-v, \lambda\right)-\sum_{u v \in E\left(D^{\prime \prime}\right)} \Phi\left(D^{\prime \prime}-u-v, \lambda\right)-2 \sum_{C^{\prime \prime} \in \mathcal{C}_{v}^{\prime \prime}} h\left(C^{\prime \prime}\right) \Phi\left(D^{\prime \prime}-C^{\prime \prime}, \lambda\right) .
$$

Note that $G-v$ is a forest. By Theorem 11, all mixed forests with the same underlying graph are switching equivalent, and therefore have the same spectrum. Thus we have

$$
\Phi\left(D^{\prime}-v, \lambda\right)=\Phi\left(D^{\prime \prime}-v, \lambda\right)
$$


and

$$
\sum_{u v \in E\left(D^{\prime}\right)} \Phi\left(D^{\prime}-u-v, \lambda\right)=\sum_{u v \in E\left(D^{\prime \prime}\right)} \Phi\left(D^{\prime \prime}-u-v, \lambda\right)
$$

Therefore,

$$
\Phi\left(D^{\prime}, \lambda\right)-\Phi\left(D^{\prime \prime}, \lambda\right)=2 \sum_{C^{\prime \prime} \in \mathcal{C}_{v}^{\prime \prime}} h\left(C^{\prime \prime}\right) \Phi\left(D^{\prime \prime}-C^{\prime \prime}, \lambda\right)-2 \sum_{C^{\prime} \in \mathcal{C}_{v}^{\prime}} h\left(C^{\prime}\right) \Phi\left(D^{\prime}-C^{\prime}, \lambda\right) .
$$

This completes the proof.

Theorem 25. A bicyclic graph $G$ is ODHS if and only if its kernel is $\theta\left(l_{1}, l_{2}, l_{3}\right)$ for some positive integers $l_{1}, l_{2}, l_{3}$ satisfying one of the following conditions:

(1) $l_{1}, l_{2}, l_{3}$ are mutually distinct.

(2) If exactly two of $l_{1}, l_{2}, l_{3}$ are equal (that is, there are exactly two cycles having the same length, say $C_{1}$ and $\left.C_{2}\right)$, then $\Phi\left(G-C_{1}\right) \neq \Phi\left(G-C_{2}\right)$ and $\Phi\left(G-C_{1}\right)-\Phi\left(G-C_{2}\right) \neq$ $2 \Phi\left(G-C_{3}\right)$.

(3) If $l_{1}=l_{2}=l_{3}$, then for any indexes $i, j, k$ with $\{i, j, k\}=\{1,2,3\}, \Phi\left(G-C_{i}\right) \neq$ $\Phi\left(G-C_{j}\right)$ and $\Phi\left(G-C_{i}\right)+\Phi\left(G-C_{j}\right) \neq 2 \Phi\left(G-C_{k}\right)$.

Proof. By Lemma 23, every bicyclic ODHS graph $G$ must contain a theta graph as its kernel, say $\theta\left(l_{1}, l_{2}, l_{3}\right)$. Denote $\mathscr{C}=\left\{C_{1}, C_{2}\right\}$ to be a strong cycle basis of $G$ and denote the third cycle of $G$ by $C_{3}$.

Without loss of generality, we assume that the directions of $C_{1}, C_{2}, C_{3}$ are coincident and $\left\|C_{1}\right\| \leqslant\left\|C_{2}\right\| \leqslant\left\|C_{3}\right\|$, where $\left\|C_{j}\right\|$ is the length of $C_{j}$ for $1 \leqslant j \leqslant 3$. It follows from Lemma 16 that the value of $C_{3}$ is the product of $C_{1}$ 's and $C_{2}$ 's for any mixed graph in $\mathcal{D}(G)$. Let $D^{\prime}$ and $D^{\prime \prime}$ be two mixed graphs in $\mathcal{D}(G)$. Applying Lemma 24, we have the following Claims. We only prove one of them as their proofs are similar.

Claim 1 If $\mathfrak{s}\left(D^{\prime}\right)=(1,-1)$ and $\mathfrak{s}\left(D^{\prime \prime}\right)=(\mathrm{i}, \mathrm{i})$, then $D^{\prime}$ is cospectral to $D^{\prime \prime}$ if and only if $\Phi\left(G-C_{1}\right)=\Phi\left(G-C_{2}\right)$.

Since the directions of $C_{1}, C_{2}, C_{3}$ are coincident, it follows from Lemma 16 that $h\left(C_{3}^{\prime}\right)=$ -1 and $h\left(C_{3}^{\prime \prime}\right)=-1$. Using Lemma 24 and assuming that $v$ is one of the common vertices of $C_{1}, C_{2}$ and $C_{3}, D^{\prime}$ is cospectral to $D^{\prime \prime}$ if and only if

$$
\Phi\left(D^{\prime}-C_{1}^{\prime}\right)-\Phi\left(D^{\prime}-C_{2}^{\prime}\right)-\Phi\left(D^{\prime}-C_{3}^{\prime}\right)=-\Phi\left(D^{\prime \prime}-C_{3}^{\prime \prime}\right) .
$$

Noting that all mixed forests with the same underlying graph have the same characteristic polynomial which is the characteristic polynomial of their underlying graph, we have $\Phi\left(D^{\prime}-C_{1}^{\prime}\right)=\Phi\left(G-C_{1}\right), \Phi\left(D^{\prime}-C_{2}^{\prime}\right)=\Phi\left(G-C_{2}\right)$ and $\Phi\left(D^{\prime}-C_{3}^{\prime}\right)=\Phi\left(D^{\prime \prime}-C_{3}^{\prime \prime}\right)$. This proves Claim 1.

Claim 2 If $\mathfrak{s}\left(D^{\prime}\right)=(1,-1)$ and $\mathfrak{s}\left(D^{\prime \prime}\right)=(\mathrm{i},-\mathrm{i})$, then $D^{\prime}$ is cospectral to $D^{\prime \prime}$ if and only if $\Phi\left(G-C_{1}\right)-\Phi\left(G-C_{2}\right)=2 \Phi\left(G-C_{3}\right)$.

Claim 3 If $\mathfrak{s}\left(D^{\prime}\right)=(1, \mathrm{i})$ and $\mathfrak{s}\left(D^{\prime \prime}\right)=( \pm \mathrm{i}, 1)$, then $D^{\prime}$ is cospectral to $D^{\prime \prime}$ if and only if $\Phi\left(G-C_{1}\right)=\Phi\left(G-C_{2}\right)$.

Claim 4 If $\mathfrak{s}\left(D^{\prime}\right)=(-1,-1)$ and $\mathfrak{s}\left(D^{\prime \prime}\right)=(\mathrm{i}, \mathrm{i})$ or $(-\mathrm{i},-\mathrm{i})$, then $D^{\prime}$ is cospectral to $D^{\prime \prime}$ if and only if $\Phi\left(G-C_{1}\right)+\Phi\left(G-C_{2}\right)=2 \Phi\left(G-C_{3}\right)$. 
To prove the necessity, it is enough to show that for any graph $G$ not satisfying the conditions (1), (2) and (3), there exist two mixed graphs $D^{\prime}$ and $D^{\prime \prime}$ in $\mathcal{D}(G)$ which are not switching equivalent, but cospectral. In fact, the Claims 1-4 imply the necessity.

To prove the sufficiency, denote $\Phi\left(D^{\prime}, \lambda\right)=\sum_{j=0}^{n} c_{j}^{\prime} \lambda^{n-j}\left(\Phi\left(D^{\prime \prime}, \lambda\right)=\sum_{j=0}^{n} c_{j}^{\prime \prime} \lambda^{n-j}\right.$, resp.) to be the characteristic polynomial of $D^{\prime}$ ( $D^{\prime \prime}$, resp.). Obviously, it is sufficient to prove that for any mixed graphs $D^{\prime}$ and $D^{\prime \prime}$ with the same underlying graph $G$ satisfying one of the conditions (1), (2) and (3), if $\mathfrak{s}\left(D^{\prime}\right)$ and $\mathfrak{s}\left(D^{\prime \prime}\right)$ are not conjugate equivalent, then $D^{\prime}$ and $D^{\prime \prime}$ are not cospectral, that is, $c_{j}^{\prime} \neq c_{j}^{\prime \prime}$ for some $j(1 \leqslant j \leqslant n)$.

Claim 5 If $\mathfrak{s}\left(D^{\prime}\right)$ and $\mathfrak{s}\left(D^{\prime \prime}\right)$ contain no real entry, then $c_{\left\|C_{3}\right\|}^{\prime} \neq c_{\left\|C_{3}\right\|}^{\prime \prime}$.

Since $\mathfrak{s}\left(D^{\prime}\right)$ and $\mathfrak{s}\left(D^{\prime \prime}\right)$ are not conjugate equivalent, without loss of generality, we assume that $\mathfrak{s}\left(D^{\prime}\right)=(\mathrm{i}, \mathrm{i})$ and $\mathfrak{s}\left(D^{\prime \prime}\right)=(\mathrm{i},-\mathrm{i})$. It follows from Lemma 16 that $\left\{h\left(C_{3}^{\prime}\right), h\left(C_{3}^{\prime \prime}\right)\right\}$ $=\{-1,1\}$. By Lemma 22, we have $c_{\left\|C_{3}\right\|}^{\prime} \neq c_{\left\|C_{3}\right\|}^{\prime \prime}$. This proves Claim 5.

Thus, we may assume that $\mathfrak{s}\left(D^{\prime}\right)$ or $\mathfrak{s}\left(D^{\prime \prime}\right)$ contains at least one real component. We divide the remaining proof into three cases according to the conditions of the theorem.

Case 1: $l_{1}, l_{2}, l_{3}$ are mutually distinct.

We can assume that $j(1 \leqslant j \leqslant 2)$ is the first index such that one of $h\left(C_{j}^{\prime}\right)$ and $h\left(C_{j}^{\prime \prime}\right)$ is real and $h\left(C_{j}^{\prime}\right) \neq h\left(C_{j}^{\prime \prime}\right)$. By Lemma $22, c_{\left\|C_{j}\right\|}^{\prime} \neq c_{\left\|C_{j}\right\|}^{\prime \prime}$, as required.

Case 2: Exactly two of $l_{1}, l_{2}, l_{3}$ are equal.

We can assume that $\left\|C_{1}\right\|=\left\|C_{2}\right\|<\left\|C_{3}\right\|$ and the number of real entries of $\mathfrak{s}\left(D^{\prime}\right)$ is at most that of $\mathfrak{s}\left(D^{\prime \prime}\right)$. Firstly, we consider $\mathfrak{s}\left(D^{\prime}\right)$ as a real vector. If $h\left(C_{1}^{\prime}\right)=h\left(C_{2}^{\prime}\right) \in\{ \pm 1\}$ and $\mathfrak{s}\left(D^{\prime}\right) \neq \mathfrak{s}\left(D^{\prime \prime}\right)$, it follows from Lemma 22 that $D^{\prime}$ and $D^{\prime \prime}$ are not cospectral. If $h\left(C_{1}^{\prime}\right)=-h\left(C_{2}^{\prime}\right) \in\{ \pm 1\}$, without loss of generality, let $\mathfrak{s}\left(D^{\prime}\right)=(1,-1)$. By Lemma 22 , only three cases need to consider that $h\left(C_{1}^{\prime \prime}\right)=h\left(C_{2}^{\prime \prime}\right) \in\{ \pm \mathrm{i}\}, h\left(C_{1}^{\prime \prime}\right)=-h\left(C_{2}^{\prime \prime}\right) \in\{ \pm \mathrm{i}\}$ and $\mathfrak{s}\left(D^{\prime \prime}\right)=(-1,1)$. For the case $h\left(C_{1}^{\prime \prime}\right)=h\left(C_{2}^{\prime \prime}\right) \in\{ \pm \mathbf{i}\}$, without loss of generality, we write $\mathfrak{s}\left(D^{\prime \prime}\right)=(\mathrm{i}, \mathrm{i})$. One can deduce that $h\left(C_{3}^{\prime}\right)=-1$ and $h\left(C_{3}^{\prime \prime}\right)=-1$ as $\mathfrak{s}\left(D^{\prime}\right)=(1,-1)$ and $\mathfrak{s}\left(D^{\prime \prime}\right)=(\mathrm{i}, \mathrm{i})$. By Claim $1, D^{\prime}$ and $D^{\prime \prime}$ are not cospectral since $\Phi\left(G-C_{1}\right) \neq \Phi\left(G-C_{2}\right)$. For the case $h\left(C_{1}^{\prime \prime}\right)=-h\left(C_{2}^{\prime \prime}\right) \in\{ \pm \mathrm{i}\}$, without loss of generality, we write $\mathfrak{s}\left(D^{\prime \prime}\right)=(\mathrm{i},-\mathrm{i})$. Hence, $h\left(C_{3}^{\prime}\right)=-1$ and $h\left(C_{3}^{\prime \prime}\right)=1$. By Claim $2, D^{\prime}$ and $D^{\prime \prime}$ are not cospectral, since $\Phi\left(G-C_{1}\right)-\Phi\left(G-C_{2}\right) \neq 2 \Phi\left(G-C_{3}\right)$. For the case $\mathfrak{s}\left(D^{\prime \prime}\right)=(-1,1), h\left(C_{3}^{\prime}\right)=-1$ and $h\left(C_{3}^{\prime \prime}\right)=-1$. It follows by a proof similar to the proof of Claim 1 that $D^{\prime}$ and $D^{\prime \prime}$ are not cospectral, since $\Phi\left(G-C_{1}\right) \neq \Phi\left(G-C_{2}\right)$.

Next, we consider the case that $\mathfrak{s}\left(D^{\prime}\right)$ exactly contains one real entry. Without loss of generality, let $\mathfrak{s}\left(D^{\prime}\right)=(1, \mathrm{i})$. We only consider the cases that $\mathfrak{s}\left(D^{\prime \prime}\right)=( \pm \mathbf{i}, 1)$. Otherwise, by Lemma 22 , it is easy to check that $D^{\prime}$ and $D^{\prime \prime}$ are not cospectral if $\mathfrak{s}\left(D^{\prime}\right)$ and $\mathfrak{s}\left(D^{\prime \prime}\right)$ are not conjugate equivalent. We have $h\left(C_{3}^{\prime}\right)=\mathrm{i}$ and $h\left(C_{3}^{\prime \prime}\right)= \pm \mathrm{i}$ from $\mathfrak{s}\left(D^{\prime}\right)=(1, \mathrm{i})$ and $\mathfrak{s}\left(D^{\prime \prime}\right)=( \pm \mathrm{i}, 1)$. Using Lemma 24 , it follows by a proof similar to the proof of Claim 1 that $D^{\prime}$ and $D^{\prime \prime}$ are not cospectral since $\Phi\left(G-C_{1}\right) \neq \Phi\left(G-C_{2}\right)$. This completes the proof of Case 2 .

Case 3: $l_{1}=l_{2}=l_{3}$.

Clearly, $\left\|C_{1}\right\|=\left\|C_{2}\right\|=\left\|C_{3}\right\|$. We also assume that the number of real components of $\mathfrak{s}\left(D^{\prime}\right)$ is at most that of $\mathfrak{s}\left(D^{\prime \prime}\right)$. Firstly, we consider $\mathfrak{s}\left(D^{\prime}\right)$ as a real vector. If $h\left(C_{1}^{\prime}\right)=$ $h\left(C_{2}^{\prime}\right)=1$ and $\mathfrak{s}\left(D^{\prime}\right) \neq \mathfrak{s}\left(D^{\prime \prime}\right)$, it follows from Lemma 22 that $D^{\prime}$ and $D^{\prime \prime}$ are not cospectral. If $h\left(C_{1}^{\prime}\right)=h\left(C_{2}^{\prime}\right)=-1$, then $h\left(C_{3}^{\prime}\right)=1$. If $\mathfrak{s}\left(D^{\prime}\right) \neq \mathfrak{s}\left(D^{\prime \prime}\right)$ and $\mathfrak{s}\left(D^{\prime \prime}\right)$ exactly contains 


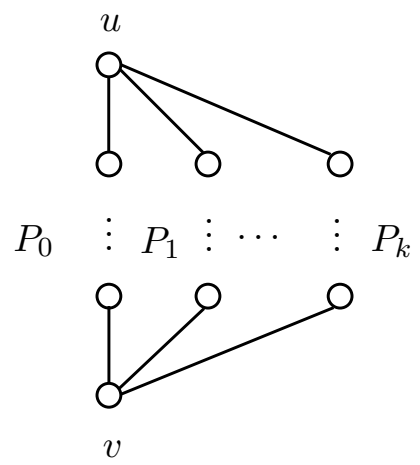

Figure 4: Graph $G\left(l_{0}, l_{1}, \ldots, l_{k}\right)$.

two real components, by Lemma $22, D^{\prime}$ and $D^{\prime \prime}$ are not cospectral, we are done. If $\mathfrak{s}\left(D^{\prime \prime}\right)$ exactly contains one real component, one can conclude that it must be -1 . Otherwise, by Lemma $22, D^{\prime}$ and $D^{\prime \prime}$ are not cospectral. Without loss of generality, write $\mathfrak{s}\left(D^{\prime \prime}\right)=$ $(-1, \pm \mathrm{i})$. It implies that $h\left(C_{3}^{\prime \prime}\right)= \pm \mathrm{i}$. Using Lemma 24 , it follows by a proof similar to the proof of Claim 1 that $D^{\prime}$ and $D^{\prime \prime}$ are not cospectral as $\Phi\left(G-C_{2}\right) \neq \Phi\left(G-C_{3}\right)$. Suppose that $\mathfrak{s}\left(D^{\prime \prime}\right)$ contains no real component. By Lemma $22, \mathfrak{s}\left(D^{\prime \prime}\right)$ must be equal to $(\mathrm{i}, \mathrm{i})$ or $(-\mathrm{i},-\mathrm{i})$. By Claim $4, D^{\prime}$ and $D^{\prime \prime}$ are not cospectral as $\Phi\left(G-C_{1}\right)+\Phi\left(G-C_{2}\right) \neq 2 \Phi\left(G-C_{3}\right)$.

Finally, we consider the case that $\mathfrak{s}\left(D^{\prime}\right)$ contains exactly one real component. Without loss of generality, we assume that $\mathfrak{s}\left(D^{\prime}\right)=(1, \mathrm{i})$. One can deduce that $h\left(C_{3}^{\prime}\right)=\mathrm{i}$. If $\mathfrak{s}\left(D^{\prime \prime}\right)$ contains exactly one real component, we only need to consider the cases that $\mathfrak{s}\left(D^{\prime \prime}\right)=$ $( \pm \mathrm{i}, 1)$. Otherwise, by Lemma 22 , it is easy to verify that $D^{\prime}$ and $D^{\prime \prime}$ are not cospectral if $\mathfrak{s}\left(D^{\prime}\right)$ and $\mathfrak{s}\left(D^{\prime \prime}\right)$ are not conjugate equivalent. We have $h\left(C_{3}^{\prime \prime}\right)= \pm \mathbf{i}$ since $\mathfrak{s}\left(D^{\prime \prime}\right)=( \pm \mathrm{i}, 1)$. By Claim $3, D^{\prime}$ and $D^{\prime \prime}$ are not cospectral if $\Phi\left(G-C_{1}\right) \neq \Phi\left(G-C_{2}\right)$. Suppose that $\mathfrak{s}\left(D^{\prime \prime}\right)$ contains exactly no real component. By Lemma $22, h\left(C_{1}^{\prime \prime}\right)=-h\left(C_{2}^{\prime \prime}\right) \in\{ \pm \mathrm{i}\}$. Using Lemma 24, it follows by a proof similar to the proof of Claim 1 that $D^{\prime}$ and $D^{\prime \prime}$ are not cospectral as $\Phi\left(G-C_{1}\right) \neq \Phi\left(G-C_{3}\right)$.

The problem which $k$-cyclic graphs with $k \geqslant 3$ are ODHS is open. In the rest of paper, we construct an infinite family of $k$-cyclic ODHS graphs. They can achieve the upper bound in Theorem 19.

Let $G\left(l_{0}, l_{1}, \ldots, l_{k}\right)$ be a graph with two given vertices $u, v$ and $k+1$ paths joining them, say $P_{0}, P_{1}, \ldots P_{k}$, where $P_{i}$ has length $l_{i}$ for all $i(0 \leqslant i \leqslant k)$, satisfying $\left\|C_{i j}\right\|<\left\|C_{i^{\prime} j^{\prime}}\right\|$ if and only if $i<i^{\prime}$, or $i=i^{\prime}, j<j^{\prime}$, where $C_{i j}$ is a cycle with length $\left\|C_{i j}\right\|$ consisting of $P_{i}$ and $P_{j}(0 \leqslant i<j \leqslant k)$. See Figure 4 . It is easy to see that $\left\{C_{01}, \ldots, C_{0 k}\right\}$ is a strong cycle basis of $G\left(l_{0}, l_{1}, \ldots, l_{k}\right)$ as $C_{i j}=C_{0 i} \oplus_{s} C_{0 j}$ for all $1 \leqslant i<j \leqslant k$. We suppose that the directions of any cycles in $G\left(l_{0}, l_{1}, \ldots, l_{k}\right)$ are clockwise. Thus $C_{0 i}, C_{0 j}$ and $C_{i j}$ are coincident for all $1 \leqslant i<j \leqslant k$.

Theorem 26. The graph $G\left(l_{0}, l_{1}, \ldots, l_{k}\right)$ defined above is ODHS and $n_{s}\left(G\left(l_{0}, l_{1}, \ldots, l_{k}\right)\right)=$ $2^{2 k-1}+2^{k-1}$.

Proof. It follows from Lemma 20 that $G\left(l_{0}, l_{1}, \ldots, l_{k}\right)$ is free with respect to the strong cycle basis $\left\{C_{01}, \ldots, C_{0 k}\right\}$, which implies that $n_{s}\left(G\left(l_{0}, l_{1}, \ldots, l_{k}\right)\right)=2^{2 k-1}+2^{k-1}$. 
Let $D^{\prime}$ and $D^{\prime \prime}$ be two mixed graphs with underlying graph $G\left(l_{0}, l_{1}, \ldots, l_{k}\right)$. In order to prove that $G\left(l_{0}, l_{1}, \ldots, l_{k}\right)$ is ODHS, it is sufficient to prove that if $\mathfrak{s}\left(D^{\prime}\right)$ and $\mathfrak{s}\left(D^{\prime \prime}\right)$ are not conjugate equivalent, then $D^{\prime}$ and $D^{\prime \prime}$ are not cospectral.

We first assume that for any $j(1 \leqslant j \leqslant k)$, either $h\left(C_{0 j}^{\prime}\right)=\overline{h\left(C_{0 j}^{\prime \prime}\right)}$ or $h\left(C_{0 j}^{\prime}\right)=$ $h\left(C_{0 j}^{\prime \prime}\right) \in\{ \pm \mathrm{i}\}$. If not, there is some $p$ such that $h\left(C_{0 p}^{\prime}\right) \neq \overline{h\left(C_{0 p}^{\prime \prime}\right)}$ and one of $h\left(C_{0 p}^{\prime}\right)$ and $h\left(C_{0 p}^{\prime \prime}\right)$ is real. Suppose that $p$ is the first index satisfying the condition. This implies that either $h\left(C_{0 j}^{\prime}\right)=\overline{h\left(C_{0 j}^{\prime \prime}\right)}$ or $h\left(C_{0 j}^{\prime}\right)=h\left(C_{0 j}^{\prime \prime}\right) \in\{ \pm \mathrm{i}\}$ for any $j(1 \leqslant j \leqslant p-1)$. It follows from Lemma 22 that $c_{j}^{\prime}=c_{j}^{\prime \prime}$ for $0 \leqslant j<l_{0}+l_{p}$ and $c_{l_{0}+l_{p}}^{\prime} \neq c_{l_{0}+l_{p}}^{\prime \prime}$, we are done.

Since $\mathfrak{s}\left(D^{\prime}\right) \neq \mathfrak{s}\left(D^{\prime \prime}\right)$ and $\mathfrak{s}\left(D^{\prime}\right) \neq \overline{\mathfrak{s}\left(D^{\prime \prime}\right)}$, we may suppose that $p$ is the first index such that $h\left(C_{0 p}^{\prime}\right) \neq \overline{h\left(C_{0 p}^{\prime \prime}\right)}$ and $q$ is the first index such that $h\left(C_{0 q}^{\prime}\right) \neq h\left(C_{0 q}^{\prime \prime}\right)$. By

the assumption mentioned above, $h\left(C_{0 p}^{\prime}\right)=h\left(C_{0 p}^{\prime \prime}\right) \in\{ \pm \mathrm{i}\}$ and $h\left(C_{0 q}^{\prime}\right)=\overline{h\left(C_{0 q}^{\prime \prime}\right)} \in\{\mathrm{i},-\mathrm{i}\}$. One can conclude that the corresponding basic cycles with length less than $l_{p}+l_{q}$ in $D^{\prime}$ and $D^{\prime \prime}$ have the same value. By Lemma $22, c_{i}^{\prime}=c_{i}^{\prime \prime}$ for $1 \leqslant i<l_{p}+l_{q}$ and $c_{l_{p}+l_{q}}^{\prime} \neq c_{l_{p}+l_{q}}^{\prime \prime}$.

By Theorem 26, $G\left(t l_{0}, t l_{1}, \ldots, t l_{k}\right)$ is ODHS for every integer $t \geqslant 1$. This implies the following result.

Corollary 27. There are infinitely many $k$-cyclic ODHS graphs for any $k \geqslant 2$.

\section{Acknowledgements}

The authors are very grateful to the referees for their valuable comments and corrections.

\section{References}

[1] J. A. Bondy and L. Lovász. Cycles through specified vertices of a graph. Combinatorica, 1:117-140, 1981.

[2] C. Chen, J. Huang, and S.-C. Li. On the relation between the H-rank of a mixed graph and the matching number of its underlying graph. Linear Multilinear Algebra, 66:1853-1869, 2018.

[3] C. Chen, S.-C. Li, and M.-J. Zhang. Relation between the H-rank of a mixed graph and the rank of its underlying graph. Discrete Math., 342:1300-1309, 2019.

[4] X.-L. Chen, X.-L. Li, and Y.-Y. Zhang. 3-regular mixed graphs with optimum Hermitian energy. Linear Algebra Appl., 496:475-486, 2016.

[5] E. R. van Dam and W. H. Haemers. Which graphs are determined by their spectrum? Linear Algebra Appl., 373:241-272, 2003.

[6] E. R. van Dam and W. H. Haemers. Developments on spectral characterization of graphs. Discrete Math., 309:576-586, 2009.

[7] G. Greaves, B. Mohar, and O. Suil. Interlacing families and the Hermitian spectral norm of digraphs. Linear Algebra Appl., 564:201-208, 2019.

[8] K. Guo and B. Mohar. Hermitian adjacency matrix of digraphs and mixed graphs. J. Graph Theory, 85:217-248, 2016. 
[9] K. Guo and B. Mohar. Digraphs with Hermitian spectral radius below 2 and their cospectrality with paths. Discrete Math., 340:2616-2632, 2017.

[10] D. Hu, X.-L. Li, X.-G. Liu, and S.-G. Zhang. The spectral distribution of random mixed graphs. Linear Algebra Appl., 519:343-365, 2017.

[11] J.-X. Liu and X.-L. Li. Hermitian-adjacency matrices and Hermitian energies of mixed graphs. Linear Algebra Appl., 466:182-207, 2015.

[12] G. J. Minty. A theorem on n-coloring the point of a linear graph. Amer. Math. Monthly, 67:623-624, 1962.

[13] B. Mohar. Hermitian adjacency spectrum and switching equivalence of mixed graphs. Linear Algebra Appl., 489:324-340, 2016.

[14] R. Steinberg and D. H. Younger. Grötzsch theorem for the projective plane. Ars Combin., 28:15-31, 1989.

[15] M. M. Sysło. On cycle bases of a graph. Networks, 9:123-132, 1979.

[16] F.-L. Tian and D. Wong. Nullity of Hermitian adjacency matrices of mixed graphs. J. Math. Res. Appl., 38:23-33, 2018.

[17] L. M. Vitaver. Finding minimal vertex coloring of a graph with Boolean power of the incidence matrix, (in Russian). Dokl. AN SSSR, 147:758-759, 1962.

[18] Y. Wang, B.-J. Yuan, S.-D. Li, and C.-J. Wang. Mixed graphs with H-rank 3. Linear Algebra Appl., 524:22-34, 2017.

[19] P. Wissing and E. R. van Dam. The negative tetrahedron and the first infinite family of connected digraphs that are strongly determined by the Hermitian spectrum. $J$. Combin. Theory Ser. A, 173:105232, 2020.

[20] G.-H. Yu and H. Qu. Hermitian Laplacian matrix and positive of mixed graphs. Appl. Math. Comput., 269:70-76, 2015.

[21] B.-J. Yuan, Y. Wang, S.-C. Gong, and Y. Qiao. On mixed graphs whose Hermitian spectral radii are at most 2. Graphs Combin., 2020. https://doi.org/10.1007/s00373-020-02181-w. 